\title{
Testing gravity with motion of satellites around galaxies: Newtonian gravity against Modified Newtonian Dynamics
}

\author{
Anatoly Klypin, ${ }^{1}$ and Francisco Prada ${ }^{2}$ \\ ${ }^{1}$ Astronomy Department, New Mexico State University, MSC 4500, P.O.Box 30001, Las Cruces, NM, \\ 880003-8001, USA \\ ${ }^{2}$ Instituto de Astrofísica de Andalucía (CSIC), Camino Bajo de Huetor, 50, E-18008 Granada, Spain
}

\begin{abstract}
The standard cosmological model is very successful on scales larger than a megaparsec. However, it suffers significant strains on smaller scales, which drive development of alternative theories of gravity such the Modified Newtonian Dynamics (MOND). The motion of satellite galaxies around normal galaxies at distances 50-500 kpc provides a sensitive test for the theories. We show that observational data from the Sloan Digital Sky Survey strongly favor the standard model: all three major statistics of satellites - the number-density profile, the line-of-sight velocity dispersion, and the distribution function of the velocities - agree remarkably well with the predictions of the standard cosmological model. We argue that the success of the standard model extends to scales (50-500) kpc, much lower than what was previously considered. MOND dramatically fails on these scales by predicting nearly constant rms velocities of satellites, while observations indicate that the rms velocities are declining with distance from the galaxy, and exclude constant velocities at a $\sim 10 \sigma$ level.
\end{abstract}

Subject headings: cosmology: theory — dark matter — galaxies: halos — galaxies: structure - methods: numerical

\section{Introduction}

One hundred years after Einstein, the theory of general relativity (GR) is still our best theory of gravity. In the framework of GR, the standard model of cosmology $(\Lambda C D M)$ provides a successful description of the Universe. In this model, the same fluctuations which give rise to the observed small variations in the temperature of the cosmic microwave background (CMB) grow under the force of gravity, and eventually form observed galaxies and other nonlinear structures such as filaments, voids, groups and clusters of galaxies. According to the model, only $\sim 4 \%$ of the density in the Universe is provided by normal baryonic matter (Spergel et al. 2006). The $\Lambda C D M$ model requires two additional components: a non-baryonic cold dark matter (CDM), which contributes about $30 \%$ of the average density of the Universe, and an even more mysterious dark energy, which makes up the rest (Spergel et al. 2006). The model is remarkably successful on scales larger than a few Megaparsecs. It predicted the amplitude and the spectrum of angular fluctuations in the $\mathrm{CMB}$ and in the distribution of galaxies (Bardeen et al. 1987; Holtzman 1989) that were later confirmed by observations (Spergel et al. 2006; Netterfield et al. 2002; Cole et al. 2005; Tegmark et al. 2004). The $\Lambda C D M$ model faces challenges on smaller scales. The most difficult ones are related with the rotation in the inner parts of spiral galaxies. It seems that the theory predicts too much dark matter inside $\sim 1 \mathrm{kpc}$ from the centers of galaxies (Moore 1994; Flores \& Primack 1994; de Blok et al. 2001). While there are some possible solutions of the problem (Rhee et al. 2004; Havashi \& Navarro 2006; Valenzuela et al. 2007), the problems on small scales are the strongest challenge the standard model has encountered. When compounded with the fact that there is no direct evidence of 
dark matter or dark energy, the current problems of the standard cosmological model have encouraged a small but growing community of physicists to propose alternative theories of gravity to avoid the need for dark matter.

This is the case for Modified Newtonian Dynamics (MOND), proposed by Milgrom (1983) to explain the rotation of galaxies without dark matter. According to MOND, the rotation curves in the outer regions of galaxies do not decline because the force of gravity is significantly stronger than for Newtonian gravity. In simple terms, if the force of gravity declines with distance as $r^{-1}$, then the balance of the centrifugal and gravity forces predicts that the rotational velocity does not depend on distance: $V_{\text {rot }}^{2} \propto G M$, where $M$ is the mass of normal baryonic matter. At early times MOND's main appeal was its simplicity: there is no need to make the assumption that the Universe is filled with particles that nobody has seen. Additional motivation came later from difficulties with explaining anomalies in the trajectories of the $\mathrm{Pi}$ oneer 10 and 11 space missions (Andreson et al. 2002).

Yet, for a long time MOND was not more than a conjecture. Only recently, Bekenstein proposed a relativistic version named tensor vector scalar theory (TeVeS) (Bekenstein 2004). This alternative theory of gravity provides a framework to make predictions of numerous important observational phenomena, which $\Lambda C D M$ has already done: the temperature fluctuations seen in the cosmic microwave background, gravitational lensing, and the large scale structure of the universe. With maturity came problems. Rotation curves of some galaxies - the initial strong argument for MOND - cannot be explained by MOND. In about $1 / 4$ of galaxies considered by proponents of MOND the predicted velocities are well above the observations in the very central regions (Sanders \& McGaugh 2002). RMS velocities of stars in some dwarf spheroidal galaxies (Lokas, Mamon, \& Prada 2006) also present problems.

So far, the most severe challenges for MOND are coming from clusters of galaxies. Dynamics of galaxies in clusters cannot be explained by MOND and requires introduction of dark matter, possibly in the form of a massive $(\sim 2 \mathrm{eV})$ neutrino (Sanders \& McGaugh 2002). We do not know whether this modification can explain some properties of clusters of galaxies such as the "Bullet Cluster", where the baryonic mass (galaxies and gas) is clearly separated from the gravitational mass, as indicated by gravitational lensing Clowe et al. 2006; Angus, Famaey, \& Zhao 2006). In any case, for MOND to survive it too must invoke dark matter and dark energy.

There is no doubt that alternative theories of gravity represent a challenge to the standard model of cosmology and GR. Any theory or model must respond to these challenges. Here we present a number of observations to test gravity and dark matter in the peripheral parts of galaxies at distances 50-500 kpc from the centers of galaxies. These scales can be tested by studying the motion of satellites of galaxies. This is a relatively old field in extragalactic astronomy and historically it was one of main arguments for the presence of dark matter (Zaritsky \& White 1994; Prada et al. 2003).

\section{Results}

We use the Sloan Digital Sky Survey (SDSS; www.sdss.org) - the largest photometric and spectroscopic astronomical survey ever undertaken of the local Universe - to study the motion of satellites. As of Data Release Four (DR4) (Adelman-McCarthy et al. 2006), imaging data are available over $6670 \mathrm{deg}^{2}$ in five photometric bands. In addition to the CCD imaging, the SDSS 2.5m telescope on Apache Point, New Mexico, measured spectra of galaxies, providing distance determinations. Approximately half million of galaxies brighter than $r=17.77$ over $4700 \mathrm{deg}^{2}$ have been targeted for spectroscopic observations as part of SDSS and are included in DR4. Redshift accuracy is better than $30 \mathrm{~km} / \mathrm{s}$ and the overall completeness is $\sim 90 \%$. For our study we compute rest frame absolute magnitudes in the g-band from the extinction-corrected apparent magnitudes assuming a $\Lambda C D M$ cosmology with a Hubble constant $h=0.7\left(H_{0}=\right.$ $100 h \mathrm{kms}^{-1} \mathrm{Mpc}^{-1}$ ). Galaxies are split into red (early-types) and blue (late-types) populations based on the bimodality observed in the $u-r$ color distribution (Baldry et al. 2004). The local minima between the peaks of the color distribution occur near $u-r=2.3$. All magnitudes and 

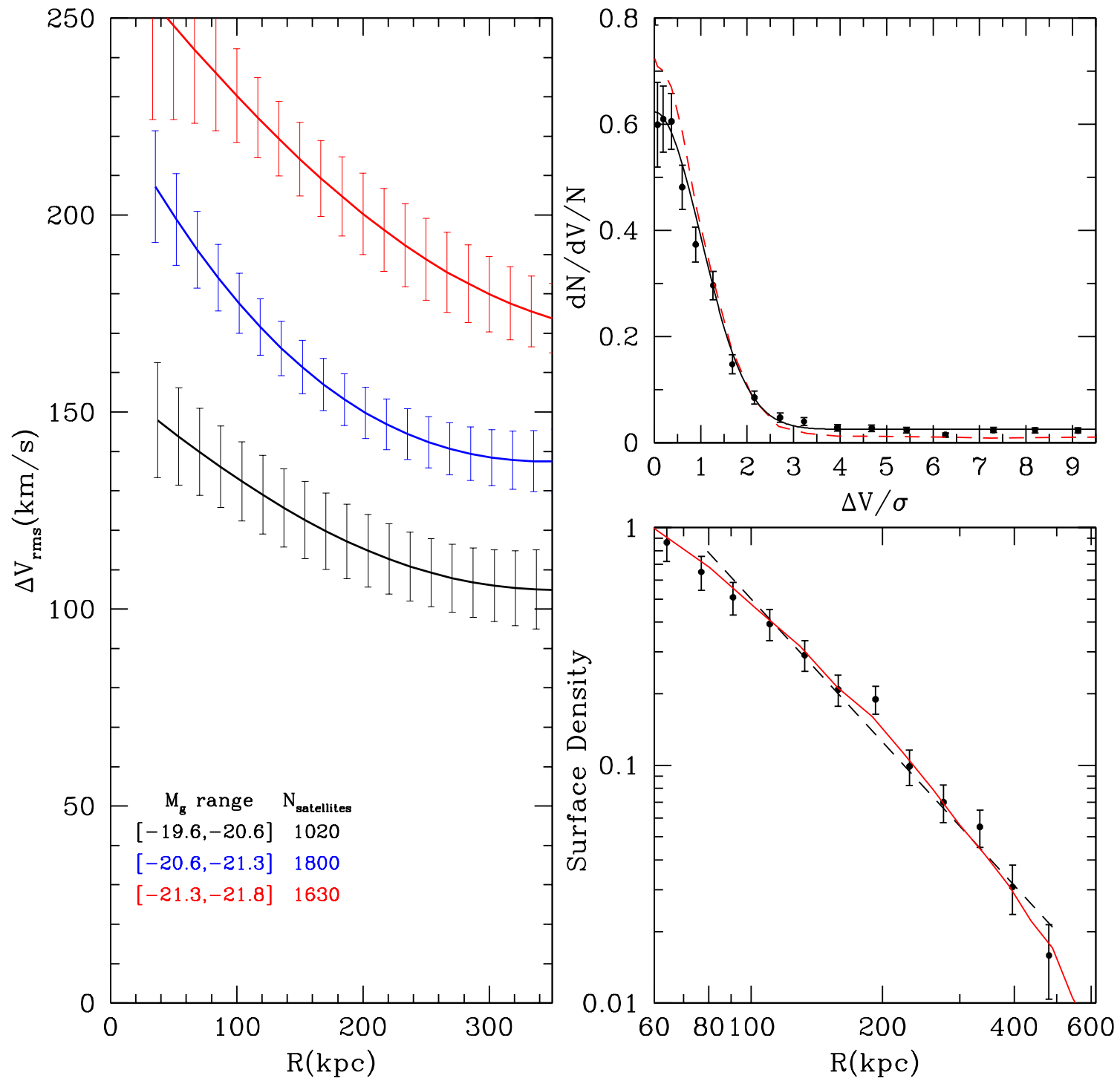

Fig. 1.- Left panel: RMS of line-of-sight velocities of satellites orbiting galaxies with absolute magnitudes indicated in the plot. Satellites move faster around more luminous galaxies. In all samples of galaxies the rms velocity is declining with distance. Right top panel: Distribution of observed line-of-sight velocities (dots with error bars) has simple structure of the Gaussian distribution with a small constant background (full curve). The background is due to objects, which in projection lie close to the central galaxy, but that are far from it in 3d space. The dashed curve is the distribution of velocities expected in the standard cosmological model drawn from $\Lambda C D M$ simulations. Right bottom panel: Surface number-density of satellites (dots with error-bars; arbitrary units) orbiting galaxies with luminosity $-20.0<M_{g}<-21.5$. The dashed line is the power law with a slope -2 . The full curve is the prediction of the $\Lambda C D M$ model.

colors are k-corrected to $z=0$. Because calculations of MOND gravity for non-spherical objects are complicated, we restrict our analysis only to red galaxies, the vast majority of which are either elliptical galaxies or are dominated by bulges. Our galaxy sample was selected from the full redshift 
sample by taking all galaxies with recession velocity $3000 \mathrm{~km} / \mathrm{s}<c z<25000 \mathrm{~km} / \mathrm{s}$. The total number of selected galaxies is about 215,000 . The SDSS heliocentric velocities were converted to the Local Group standard of rest before computing distances. We select our host galaxies as galaxies with absolute g-band magnitude brighter than $M_{g}=-19.0$ and isolated: a galaxy must be at least 4 times brighter than any other galaxy within a projected distance $R<700 \mathrm{kpc}$ and a line-of-sight velocity difference $\Delta V<1000 \mathrm{~km} / \mathrm{s}$. We define satellites as all galaxies being at least 4 times fainter than their hosts and found within a projected distance $R<1000 \mathrm{kpc}$ and velocity difference $\Delta V<1500 \mathrm{~km} / \mathrm{s}$ with respect to their hosts. Typically we find about 1.5 satellites per host. In total we have 6100 satellites with a mean luminosity of about $M_{g}=-18.0$. We bin the host galaxies by luminosity and collect information about the distribution of relative velocities $\Delta V$ and the number of satellites as the function of projected distance $R$.

Figure 1 presents the observational results. The distribution of line-of-sight velocities (top right panel) clearly shows a two-component structure: a homogeneous background of interloper galaxies (dwarfs which happen to lie along the line-of-sight, with large physical distances from the host galaxies but small projected and velocity differences, but which are not associated with the host) and a nearly Gaussian component. The surface density of the satellites also shows the same structure: at large separations the number density goes to a constant due to interlopers. We subtract the background and plot the surface density of satellites in the bottom right panel. In order to study the velocity dispersion of satellites $\Delta V_{\mathrm{rms}}^{2} \equiv\left\langle\Delta V^{2}\right\rangle$ as a function of projected distance $R$ we use a maximum likelihood method to approximate the number of satellites $N(R, \Delta V)$ per unit projected area and per unit velocity difference $\Delta V$ using an 11parameter function in the form of a constant plus a Gaussian distribution with variable velocity dispersion and normalization:

$N(R, \Delta V)=n(R) \exp \left[-V^{2} / 2 \sigma^{2}(R)\right] / \sqrt{2 \pi} \sigma+n_{0}$,

where the surface number-density of satellites $n(R)$ and the rms line-of-sight velocity $\sigma=$ $\Delta V_{\text {rms }}(R)$ are 4 th order shifted Chebyshev polynomials of the first kind. The parameter $n_{0}$ is a con- stant representing the background of interlopers. The left panel in Figure 1 shows the resulting rms velocity of satellites for three magnitude bins. In order to estimate statistical uncertainties, we run Monte Carlo simulations using the same number of hosts and satellites as in the corresponding magnitude bin in Figure 1. Note that the data points are correlated. We use Monte Carlo simulations to test statistical significance that the observed velocities are declining with distance: data for each magnitude bin reject a constant $\Delta V_{\text {rms }}(R)$ at about $3 \sigma$ confidence level. We also studied blue galaxies and find declining velocities for them. These results are in agreement with previous estimates (Prada et al. 2003; Conroy et al. 2007), but we now can exclude constant rms velocities much more reliably.

In order to compare observational results with the $\Lambda C D M$ predictions, we use high-resolution cosmological N-body simulations (Prada et al. 2006) and "observe them. Results presented in Figures 1-2 show that all three characteristics of observed satellites are reproduced by the model. Predictions for MOND can be made only by solving the Jeans equation for spherical systems, which gives the radial velocity dispersion $\sigma(r)$. In our case the Jeans equation can be written in the form:

$$
\frac{d \sigma^{2}}{d r}+\sigma^{2} \frac{(2 \beta+\alpha)}{r}=-g(r), \quad \alpha \equiv d \ln \rho / d \ln r,
$$

where $\beta(r)=1-\sigma_{\perp}^{2} / 2 \sigma^{2}$ is the velocity anisotropy. In the case of Newtonian gravity the acceleration $g=g_{N}(r)=G M(r) / r^{2}$, where the mass $M(r)$ includes both normal baryonic mass and dark matter. For MOND the acceleration $g_{\text {MOND }}$ is given by the solution of the non-linear equation

$$
g_{\mathrm{MOND}} \mu\left(\left|g_{\mathrm{MOND}}\right| / a_{0}\right)=G M_{*}(r) / r^{2},
$$

where $M_{*}$ is the mass of only baryons and $a_{0}=$ $1.2 \times 10^{-8} \mathrm{~cm} \mathrm{sec}^{-2}$. The function $\mu(x)$ can have different shapes. We used originally proposed form (Milgrom 1983) $\mu(x)=x / \sqrt{1+x^{2}}$, but also tried and accepted a newer version Famaev \& Binnev 2005) $\mu(x)=x /(1+x)$, which gives slightly better results. The value of $\alpha$ is limited by the observations presented in Figure 1: $\alpha=-(2.5-3)$. The velocity anisotropy $\beta$ is a free function because MOND does not make any predictions for $\beta$. There are constraints: asymptotically $\beta$ goes to zero at large distances and it should be a smooth 


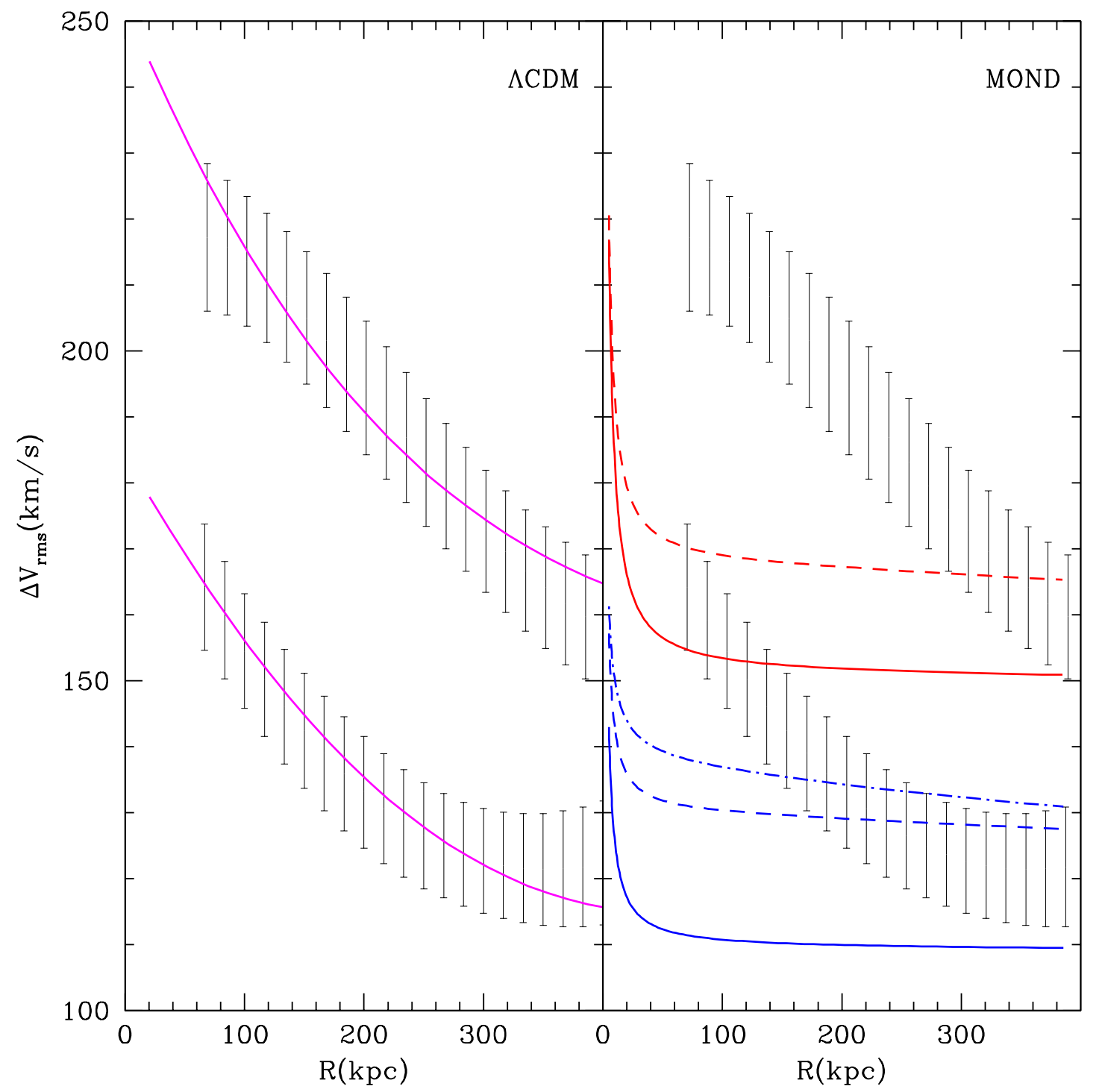

Fig. 2.- The line-of-sight velocities of galaxies in two luminosity ranges: $-20.5<M_{g}<-21.1$ (1300 satellites) and $-21.1<M_{g}<-21.6$ (1740 satellites). The vertical lines indicate $68 \%$ confidence levels. The left panel shows predictions (full curves) of the standard cosmological model for galaxies hosted by dark matter halos with maximum circular velocities of $\sim 350 \mathrm{~km} / \mathrm{s}$ (top curve) and $\sim 270 \mathrm{~km} / \mathrm{s}$ (bottom curve). The right panel shows MOND predictions. The three bottom curves are for stellar masses $8 \times 10^{10} M_{\odot}$ estimated for galaxies with $M_{g}=[-20.5,-21.1]$. The full curve is for models with isotropic velocities $\beta=0$ and with the observed slope $\alpha=-3$ of the 3D number density of the satellites. The curve was so much below the observed data points that for other models we decided to use the slope $\alpha=-2.5$, which is marginally compatible with the observations, but which gives larger velocities for MOND models. The dashed curve is for orbits, which are preferentially radial $(\beta=$ const $=0.5)$. The dot dashed curve is for radial orbits with changing anisotropy parameter $\beta \approx 0.5-1$. The two top curves are for the stellar mass $2 \times 10^{11} M_{\odot}$ (appropriate for galaxies in the magnitude range) and for $\beta=0$ (full curve) and $\beta=0.6$ (dashed curve). The $\Lambda C D M$ makes quite reasonable predictions, while MOND is clearly not compatible with observations. 
function. Using different values for $\beta$, we solve the equations numerically and then integrate the solution along the line-of-sight to get a prediction for $\Delta V_{\text {rms }}$. Figure 2 presents results for different magnitude ranges and for different parameters $\beta$ and $\alpha$.

\section{Conclusions}

Observational data from the Sloan Digital Sky Survey strongly favor the standard model: all three major statistics of satellites - the numberdensity profile, the line-of-sight velocity dispersion, and the distribution function of the velocities - agree remarkably well with the predictions of the standard cosmological model. Thus, the success of the standard model extends to scales (50-500) kpc, much lower than what was previously considered.

MOND fails badly in all cases by producing sharply declining velocities at small distances followed by nearly flat velocities at large distances just the opposite of what is observed in real galaxies. It is easy to understand why this happens. At large distances the Newtonian acceleration is very small and the acceleration is strongly dominated by the MOND correction: $g_{\text {MOND }} \propto 1 / r$. In this case, the solution of eq.(1) gives $\sigma=$ const for any constant value $\beta$ and $\alpha$. In more general case of non-constant $\beta$, a combination of projection effects, large and nearly constant $\alpha$, make it nearly impossible to get away from nearly constant $\Delta V_{\text {rms }}$.

\section{REFERENCES}

Spergel, D. N., et al., 2006, ArXiv Astrophysics e-prints, arXiv:astro-ph/0603449

Bardeen, J. M., Bond, J. R., Efstathiou, G., 1987, Astrophys. J., 321, 28

Holtzman, J., 1989, Astrophys. J. Supp. Series, 71,1

Netterfield, C. B., et al., 2002, Astrophys. J., 571, 604

Cole, S., et al., 2005, MNRAS, 362, 505

Tegmark, M., et al., 2004, Astrophys. J. 606, 702

Moore, B., 1994, Nature 370, 629
Flores, R. A., \& Primack, J. R., 1994, Astrophys. J. Letters, 427, L1

de Blok, W. J. G., McGaugh, S. S., Rubin, V. C., 2001, Astron. J., 122, 2396

Rhee, G., Valenzuela, O., Klypin, A., Holtzman, J., Moorthy, B., 2004, Astrophys. J., 617, 1059

Hayashi, E., \& Navarro, J. F., 2006, MNRAS, 373, 1117

Valenzuela, O., Rhee, G., Klypin, A., Governato, F., Stinson, G., Quinn, T., \& Wadsley, J., 2007, Astrophys. J. 657, 773

Milgom, M., 1983, Astrophys. J., 270, 365

Anderson et al., J. A., 2002, Phys. Rev. D, 65, 082004

Bekenstein, J. D., 2004 Phys.Rev. D, 70, 083509

Sanders, R. H. \& McGaugh, S. S., 2002, Ann.Rev.Astron. \& Astrophys 40, 263

Łokas, E., Mamon, G., Prada, F., 2006, EAS Publications Series, 20, 113

Clowe, D., et al., 2006, Astrophys. J. Letter 648, L109

Angus, G. W., Famaey, B., Zhao, H. S., 2006, MNRAS, 371, 138

Zaritsky, D., \& White, S. D. M., 1994, Astrophys. J., 435,599

Prada et al., F., 2003, Astrophys. J., 598, 260

Adelman-McCarthy, J. K., et al., 2006, Astrophys. J. Supp. Series, 162, 38

Baldry, I. K., et al., 2004, Astrophys. J., 600, 681

Conroy, C., et al., 2007, Astrophys. J., 654, 153

Prada, F., it et al., 2006, Astrophys. J., 645, 1001

Famaey, B., \& Binney, J., 2005, MNRAS, 363, 603

This 2-column preprint was prepared with the AAS LATEX macros v5.2. 


\section{Acknowledgments}

We thank S. McGaugh for challenging us to do MONDian analysis of the satellite motion. We are gratefull to J. Holtzman for numerous comments and suggestions and thank J. Betancort-Rijo for comments. We acknowledge support by the NSF grant AST-0407072 to NMSU and thank the Spanish MEC under grant PNAYA 2005-07789 for their support. Computer simulations used in this research were conducted on the Columbia supercomputer at the NASA Advanced Supercomputing Division and on Seaborg at the National Energy Research Scientific Computing Center (NERSC). 